\title{
Agronegocios
}

\section{Riesgo operativo en sistemas de producción agrícola. Propuesta base para su evaluación}

Operational risk agricultural production system. Basic proposal for its evaluation

Diego Fernando Quirós Badilla ${ }^{1}$

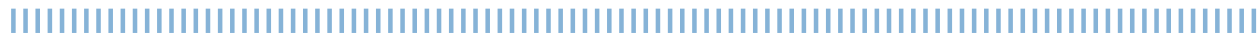

Fecha de recepción: 31 de marzo, 2019 Fecha de aprobación: 20 de junio, 2019

Vol.5 N² Julio- diciembre 2019

Quirós Badilla, D. (2019). Riesgo operativo en sistemas de producción agricola. Propuesta base para su evaluación. Revista E-Agronegocios, 5(2) https://doi.org/10.18845/rea.v5i2.4455.

DOI: https://doi.org/10.18845/rea.v5i2.4455 


\section{Resumen}

El objetivo del presente documento es incorporar la gestión administrativa de los riesgos que afectan al sector agrícola en el ámbito operativo, siendo fundamental su análisis desde los procesos productivos, la gestión del recurso humano, el manejo y uso de las tecnologías de producción, así como, los factores ambientales que generan vulnerabilidad en los cultivos. Se define de manera amplia qué factores dentro de estos aspectos de la producción pueden integrarse para evaluar sus riesgos. Por otra parte, se introduce a la gestión operativa de riesgos en sistemas agricolas una metodología de evaluación base (matrices de calor), utilizada en otros campos de la economía, que analiza la frecuencia por el impacto de un evento como el riesgo de un fenómeno o amenaza específica, y lo relaciona con la gestión (exposición) para administrar este riesgo, cuyo fin es que pueda facilitar la identificación, valoración y priorización de estos peligros, con el fin de disminuir, mitigar o transferir dichas amenazas.

Palabras clave: Riesgo, agricultura, vulnerabilidad, factores de riesgo.

\section{Abstract}

The objective of this document is to incorporate the administrative management of the risks that affect the agricultural sector in the operative field, being fundamental its analysis from the productive processes, the management of the human resource and the production technologies, as well as, the environmental factors that generate vulnerability in crops. It is broadly defined what factors within these aspects of production can be integrated to assess their risks. Moreover, a methodology of base evaluation (heat matrices), used in other fields of the economy is introduced, which analyzes the frequency by the impact of an event as the risk of a phenomenon, and relates it to the management (exposure) to manage this risk, whose purpose is to facilitate the identification, assessment and prioritization of these hazards, in order to reduce, mitigate or transfer these threats.

Key words: Risk, agriculture, 4, risk factors. 


\section{Introducción}

Las variaciones en los diversos factores que afectan la producción agrícola dentro del sistema, componen el riesgo operativo, estos factores responden al nivel de adaptación de la finca ante las variaciones climáticas y la correcta adecuación del cultivo con su entorno. Por otro lado, también toma en cuenta el uso de las técnicas adecuadas de producción y el buen manejo de los insumos (Guerra, 2002, p.165). Estos aspectos definen el correcto funcionamiento de los procesos que debe seguir el productor, sin embargo, existe una alta incertidumbre, ya que estas variaciones son difíciles de medir y de evaluar por parte de agricultores a la hora de tomar decisiones.

Otro factor a tomar en cuenta es el humano, que suele describirse como una fuente de riesgo adicional separada de la integralidad de los riesgos en la operación. La mano de obra en el sector agropecuario es critica, dado que la mayoria de las actividades agricolas son intensivas en el uso de mano de obra, en Costa Rica para el año 2017 representaba el 12,3\% de la población económicamente activa de acuerdo con la Secretaría Ejecutiva de Planificación Sectorial Agropecuaria (SEPSA, 2019).

Así mismo se toman en cuentan otros factores, como los desastres naturales y el entorno económico, político y legal. Los desastres naturales de acuerdo con la Food and Agriculture Organization (FAO, 2018) entre 2005 y 2015 representaron pérdidas de 96000 millones de dólares de daños a producción agrícolas y pecuarias. Políticas, programas sociales y de reactivación económica que no generen un ambiente apropiado al desarrollo agrícola generan inestabilidad en los productores y delimitan su crecimiento (FAO, 2003).

Existe escasa literatura sobre metodologías que midan y determinen el nivel de riesgo operativo que presenta un productor. Los sistemas de evaluación de riesgo en general van enfocados a otros tipos de riesgos, como lo son el de mercado y el financiero, donde ya existen metodologías apropiadas para su evaluación. Se desarrolla un referente teórico del concepto de riesgo y el riesgo operativo, y como este último se delimita de manera exclusiva para ámbitos como el financiero y empresarial. En el tercer apartado se realiza la clasificación de factores de riesgo operativo presentes en sistemas de producción agrícola, asi como un método base de evaluación, utilizado en otros ámbitos cuando no se posee información histórica de eventos o amenazas.

\section{Referente teórico}

\section{Concepto de riesgo y riesgo en la agricultura}

El riesgo es la posibilidad de sufrir pérdidas, que afecten el bienestar de un individuo o grupo de personas, asociado a una probabilidad de ocurrencia tanto empírica como calculada, con base a la frecuencia de eventos estudiados (Girdžiūtè, 2012; Hardaker, Lien, Anderson, \& Huirne, 2015; Harwood, Heifner, Coble, Perry, \& Somwaru, 1999); por su parte Miller, Dobbins, Pritchett, Boehlje, \& Ehmke (2004, p. 1) mencionan que el riesgo se refiere a la posibilidad que 
ocurra un evento adverso, peligro o una pérdida.

La incertidumbre se presenta cuando un agente no conoce todos los efectos negativos o daños que puede ocasionar un evento o circunstancia, en contra posición al riesgo donde se conoce todos los posibles impactos, sin embargo, su relación especifica es que cada agente toma decisiones con información incompleta (Engler, 2009, p. 13-14). El riesgo puede clasificarse de acuerdo con la categorización propuesta por Baquet, Hambleton, \& Jose, D. (1997) en riesgo productivo, riesgo de mercado o comercial, riesgo financiero, riesgo humano y riesgo legal.

Así, de acuerdo con Hansson y Lagerkvist (2012, p.737) el riesgo en la agricultura se origina dentro de diversas áreas donde se incluye la salud familiar, la legislación, las etapas productivas, el comercio, las finanzas del agricultor y los efectos de variables ambientales, en concordancia con Kahan (2008, p. 2), que menciona que el ambiente, la incidencia de plagas, las averías del equipo, las fluctuaciones de mercado, las políticas públicas y la salud de las personas involucradas en la producción, son también factores que generan riesgo.

\section{Concepto de riesgo operativo}

El término de riesgo operativo nace en el marco del funcionamiento de las instituciones financieras, donde la principal preocupación en cuanto a la cuantificación del riesgo se ha convertido en una de las necesidades centrales de los investigadores y operadores en finanzas, en cuyo caso se han propuesto modelos de cuantificación y medición del mismo, estos van desde la ejecución de modelos de indicadores básicos (Arbeláez et al, 2006; Delfiner \& Pailhé, 2009; Pacheco, 2009; Mora \& Gudiño; 2010), métodos normales de medición (Arbeláez et al, 2006; Delfiner \& Pailhé, 2009;: Mora \& Gudiño; 2010 Pacheco, 2009), y modelos de medición avanzados, utilizando criterios cuantitativos y cualitativos (Arbeláez et al., 2006; Delfiner \& Pailhé, 2009; Mora \& Gudiño, 2010; Mora, 2014; Pacheco, 2009). Es decir, la gama de estudios sobre como abarcar el tema de riesgo operativo en entidades financieras es amplia, sin embargo, es importante determinar que método dentro de esta gama que ya se utiliza en otros sectores, puede ser apropiado a utilizar en el sector agrícola.

En el contexto de las entidades financieras el concepto definido por Basilea II (segundo acuerdo del Comité Basilea de Supervisión Bancaria) para riesgo operativo hace énfasis en "el riesgo de sufrir pérdidas debido a la inadecuación o a fallos en los procesos, personal y sistemas internos o bien por causa de eventos externos" (Pacheco, 2009, p. 5); por su parte Arbeláez et al (2006, p. 98) destacan que entre los factores asociados al riesgo operacional de servicios de intermediación financiera se encuentran el "fraude interno, fraude externo, daños en activos físicos, prácticas de los empleados, prácticas del negocio, interrupción del negocio, gestión del proceso".

Así mismo, actualmente se ha ampliado la ejecución de modelos de evaluación de riesgo operativo a nivel empresarial, con la introducción de la norma ISO 31000, que abarca una serie

de alter nativas para gestionar y administrar el riesgo en las empresas, sin embargo, su ámbito de aplicación requiere amplios recursos financieros, siendo de difícil acceso para productores 
agrícolas medianos y pequeños.

El concepto de riesgo operativo como tal en sistemas de producción agrícola no está ampliamente

desarrollado, dado que la mayoría de investigaciones se enfocan en evaluar de manera general los diferentes riesgos que se presenta este sector, abarcando de manera individual los factores ambientales, productivos, financieros y de mercado, obsérvese (Hardaker et al., 2015; Harwood et al., 1999; Just \& Pope, 2003; Miller et al., 2004; Mishra \& Lence, 2005).

De manera particular se ha abarcado el concepto de riesgo en la producción para agricultura, asimilando la definición planteada dentro del contexto financiero y empresarial, pero sin incluir las variables que estas organizaciones toman en cuenta de manera conjunta. La Organización para la Cooperación y el Desarrollo Económicos (OECD, 2009, p. 16) citando a Miller et al (2004), Musser y Patrick (2002), Harwood et al (1999) y a Baquet et al (1997), destaca que el riesgo en las operaciones de un sistema productivo agrícola representa la pérdida o disminución de los rendimientos debido a factores climáticos y a plagas y enfermedades. Menciona además el peligro de no contar con mano de obra suficiente para las labores de producción como un factor de riesgo separado, lo mismo con errores en las tecnologías productivas.

En este contexto es necesario contemplar los factores endógenos que representan riesgos en la producción, así como aquellos elementos exógenos que originen vulnerabilidad de manera inmediata o directa sobre el sistema productivo, como lo son los factores ambientales. La Comisión Económica para América Latina y el Caribe (CEPAL, 2013, p. 19) citando a Cano (2006) menciona que la "amenaza es un evento natural, sociocultural o antropogénico que por su magnitud y caracteristicas pueden causar daño", haciendo alusión a que el riesgo se puede originar dentro del sistema productivo, así como provenir de factores externos, siendo vital incorporar un sistema de gestión integral de riesgo que analice estás variables.

\section{Resultados y discusión}

\section{Clasificación de factores de riesgo operativo en agricultura}

Según Quirós \& Solórzano (2018) el sector agrícola se encuentra expuesto en gran medida a diversos eventos que generan vulnerabilidad, siendo parte de estos eventos de orden macroeconómico, asi como los factores de riesgo directamente relacionados con el sector agrícola. En esta sección se describen los riesgos presentes en los procesos productivos agrícolas, en la gestión del recurso humano, la gestión de las tecnologías para la producción y los riesgos derivados de los factores climáticos y ambientales; de acuerdo a los descrito por Quirós y Solórzano (2018) y ampliado por otros autores, siguiendo el concepto general de riesgo operativo utilizado en el sector financiero y empresarial, para delimitarlos y caracterizarlos de la siguiente manera:

\section{Riesgos en los procesos de producción agricola}


El proceso que sigue un sistema de producción agrícola, sea cual sea su modalidad de producción, puede generalizarse tomando en cuenta las pautas y procedimientos establecidos por las normas de buenas prácticas agrícolas (Quirós \& Solórzano, 2018), con el ideal de que el productor posea un correcto seguimiento de los procesos en el manejo productivo, se propone identificar los riesgos en la siguiente línea de proceso:

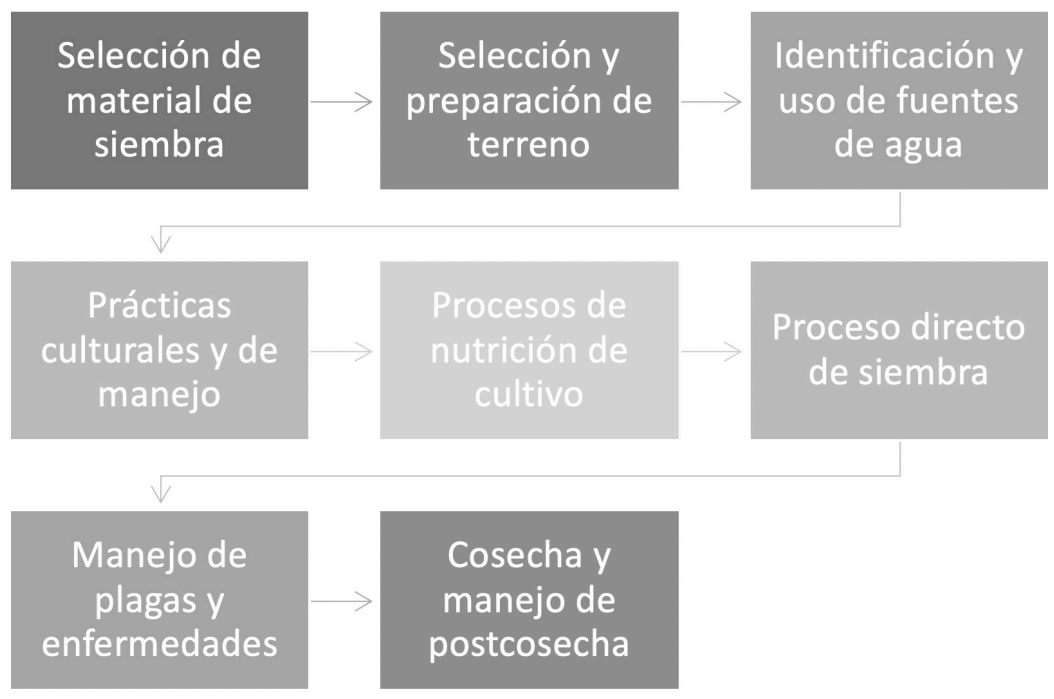

Figura 1. Línea de proceso en la producción agricola

Fuente: Modificado de Quirós y Solórzano (2018).

En cada etapa de proceso se pueden presentar situaciones recurrentes o esporádicas que generan vulnerabilidad, la identificación de las que represente mayores amenazas es necesaria y con ello su evaluación, para posteriormente gestionar una adecuada administración de estos eventos.

\section{Riesgo en el recurso humano}

De acuerdo con Kahan (2013, p. 8) el riesgo humano se refiere a los riesgos causados por enfermedad o muerte y la situación personal de la familia en la finca.; parte de estos riesgos pueden derivarse en los accidentes, la enfermedad y la muerte tanto de colaboradores como integrantes propietarios del sistema productivo, que pueden interrumpir el proceso productivo.

Por otro lado, parte de estos riesgos se reflejan en los objetivos cambiantes de las personas involucradas en la empresa agrícola que puede tener efectos tanto en el corto y largo plazo y afectar el rendimiento de la operación (Harwood et al., 1999, p. 7). Dado lo anterior es necesario determinar los factores que componen el riesgo presente en la gestión del personal, para lo cual se establecen los siguientes de acuerdo con lo propuesto por Quirós y Solórzano (2018): 
Salud: La salud del recurso humano juega un papel fundamental en un sistema de producción agrícola, se deben evaluar que eventos pueden perjudicar la salud del personal, así como cuáles pueden provocar accidentes que puedan dañar la integridad fisica de las personas que laboran en el cultivo, con el fin de poder establecer planes o medidas de mitigación para reducir un efecto no deseado en los rendimientos esperados.

Capacitación: El conocimiento de la forma correcta de cómo se realizan los procesos es fundamental para garantizar el éxito productivo, es necesario por lo tanto identificar y evaluar el daño provocado en el cultivo o bajas en los rendimientos, ocasionado por falta de conocimiento en las labores productivas.

Disponibilidad: Contar con recurso humano para la ejecución de las labores productivas es crítico, principalmente para aquellos sistemas intensivos en uso de mano de obra, o para actividades primordiales como la cosecha; la planeación de los requerimiento en recurso humano suele convertirse en un factor de riesgo cuando no se gestiona de manera apropiada.

Compromiso: Los beneficios y reconocimientos que proporcione cada empresario agrícola a las personas que laboran en la producción pueden determinarse como un factor de riesgo importante, especialmente cuando no se cuenta con suficiente mano de obra calificada. Así mismo, se debe evaluar el compromiso de los trabajadores con la producción para gestionar salidas de personas no planificadas.

\section{Riesgo en la gestión de las tecnologías de producción}

La tecnología es un elemento que genera incertidumbre, la misma surge del constante desarrollo y adopción de nuevos técnicas o métodos de producción, el desarrollo de nuevos paquetes tecnológicos, de nueva maquinaria para preparar el terreno, de sistemas de monitoreo vía GPS, de equipo de cosecha, entre otras herramientas (Miller et al., 2004; Quirós y Solórzano, 2018).

Dado lo mencionado anteriormente se describen los siguientes factores dentro de los riesgos de la gestión de la tecnología, mencionados por Quirós y Solórzano (2018):

Acceso a la tecnología: Es necesario determinar cuáles tecnologías de producción es necesario acceder para gestionar o combatir determinar eventos, en términos de nutrición un paquete tecnológico adecuado al cultivo en fundamental para estimar éxito en la producción, por otra parte, el acceso a otras tecnologías especialmente para controlar plagas y enfermedades es crítico para el desarrollo de los cultivos.

Manejo de la tecnología: Se debe poseer la capacitación de uso y manejo de las diferentes tecnologías de producción con el fin de utilizarlas de manera que su efecto deseado proporcione máximo beneficio posible sobre el cultivo. El uso incorrecto puede representar perdidas en la producción.

Mantenimiento de la tecnología: Toda tecnología que se obtenga se le debe realizar el debido mantenimiento, específicamente en el uso de equipos para realizar las labores productivas, poseer tecnologías que no se puedan utilizar por un mantenimiento inadecuado o bien un 
mal almacenamiento, puede generar contratiempos que afecten los rendimientos del cultivo.

\section{Riesgos de los factores ambientales}

El ambiente es el principal factor de dependencia para los sistemas de producción agrícola, así como a su adaptación a las variables climáticas propias de las regiones donde se establecen, sumando efectos relacionados con el cambio climático, y desastres naturales que generan un impacto en ocasiones crítico en las plantaciones. Dichos eventos no se pueden prevenir en la mayoría de los casos, y su impacto puede ser de difícil gestión, pero de acuerdo con Quirós y Solórzano (2018) se pueden gestionar acciones para disminuir el impacto o bien transferir dicho riesgos a terceros, los factores climáticos y ambientales se pueden clasificar de la siguiente manera:

Factores climáticos de la zona: Se deben identificar los principales fenómenos climáticos recurrentes en la zona y que generan de una forma u otros efectos perjudiciales en el cultivo para analizar la vulnerabilidad en la producción, y determinar planes de mitigación o adaptación a estos eventos (Quirós \& Solórzano, 2018). Su principal justificación es la exposición alta a riesgos climáticos que enfrentan los agricultores y, siendo parte de la problemática fundamental a una falta de medidas de gestión por parte de los productores (Pelka, Musshoff, \& Weber, 2015).

Desastres y catástrofes naturales: De acuerdo con Quirós \& Solórzano (2018, p. 66) "son eventos dispersos cuyas probabilidades de ocurrencia no se conocen con certeza y suelen presentarse sin previo aviso o con muy poco tiempo de reacción, es decir, su frecuencia es rara, pero su impacto suele ser importante en la producción", mayormente ampliado por Ullah et al (2015, p. 271) que consideran para su estudio como riesgos catastróficos los ocasionados por inundaciones, por lluvias fuertes, inclusive los derivados de plagas y enfermedades dado su nivel de impacto; sin embargo, su daño aunque pueda resultar masivo sobre el cultivo, puede ser soportado por medio de seguros o acceso a subvenciones estatales.

Cambio climático: Una de las mayores afectaciones en la agricultura está ligada al cambio climático, muchas regiones en diferentes países están experimentando cambios significativos de temperatura, precipitaciones, periodos de sequia, inviernos, o afectación de desastres de origen meteorológico donde antes no eran comunes (Quirós y Solórzano, 2018). Siendo a su vez un detonante para el aumento de los diversos riesgos en el sector agrícola, ya que conforme pase el tiempo su efecto puede ser mayor en las producciones (Odening \& Shen, 2014).

Pérdida de la biodiversidad: De acuerdo con Quirós y Solórzano (2018, p.69) "la agricultura es el producto de la interacción humana con la diversidad biológica a lo largo de su historia, por lo que la dependencia de la misma con la biodiversidad es indispensable para garantizar la continuidad de los sistemas de producción". Se deben considerar las afectaciones de las plantaciones sobre la biodiversidad y generar acciones para compensar dichos efectos (Seaton \& Barea, 2013, p. 25). 


\section{Evaluación por medio de matrices de calor en riesgo operativo}

Una forma para evaluar estos eventos de manera empírica en términos de riesgo es por medio de las matrices de calor (Fahad et al., 2018; Girdžiūtè, 2012; Quirós \& Solórzano, 2018; Ullah et al., 2015), también conocidas en el ámbito financiero y empresarial como matrices de evaluación de riesgo operativo, ya que utilizan el criterio de experto o bien conocimiento empírico para evaluar cada fenómeno, sin utilizar un histórico de datos para analizar cada evento. Un ejemplo claro de aplicación en el ámbito financiero de esta herramienta la presenta Palma (2011), especificando que la evaluación se debe realizar de manera integral identificando la frecuencia de eventos multiplicado por su impacto, y asociarlo con la gestión realizada para administrarlos.

Con base en los factores anteriormente clasificados para delimitar el riesgo operacional en la agricultura, es posible tropicalizar esta metodología y adaptarla para una evaluación empírica y simple por parte de los productores o evaluadores del sector agrícola.

Para la evaluación de cada factor de riesgo identificado es necesario valorar el grado de frecuencia e impacto que presenta el sistema productivo para cada evento identificado, y definir el grado de exposición (gestión) que presenta el productor ante tal acontecimiento, dependiendo de la complejidad de la evaluación, así como el número de eventos identificados. Se debe decidir si se realiza la evaluación por cada uno de los eventos identificado en cada factor, o bien tomando en cuenta los promedios obtenido en cada factor.

Se propone el uso de una escala Likert (de 1 a 5 , sin embargo, dicha escala puede cambiar de acuerdo a las condiciones y complejidad del cultivo), que justifica el uso de estos métodos dado la falta de un sistema de datos que proporcione medidas estadísticas para determinar probabilidades con cada evento identificado.

Es necesario como se mencionó anteriormente el criterio de experto del evaluador o evaluadores, ya que esto define qué tan pertinentes son las puntuaciones establecidas.

Las clasificaciones se deben realizar de acuerdo a la naturaleza del cultivo a evaluar, tomando en cuenta su ciclo de vida, tiempos de desarrollo, así como los años de experiencia del productor, con el fin de determinar con mayor precisión cada evento y su grado o probabilidad, así sea subjetiva de presentarse en el cultivo, su posible impacto y gestión establecida por el agricultor, con base en los parámetros generales de los cuadros 1, 2 y 3, que se pueden adaptar a las realidades de cada cultivo.

La frecuencia se define como la probabilidad de ocurrencia de determinado fenómeno que pueda causar daño dentro del sistema productivo, representa el grado en el cual el riesgo se puede materializar o se ha materializado en el pasado; el impacto por otro lado, es el grado de afectación que puede ocasionar ese evento una vez materializado el riesgo dentro del sistema. 
Cuadro 1. Clasificación de la frecuencia de eventos en sistemas de producción agrícola.

\begin{tabular}{ccc}
$\begin{array}{c}\text { Grado de } \\
\text { frecuencia }\end{array}$ & $\begin{array}{c}\text { Valor } \\
\text { representado }\end{array}$ & Descripción de la medición \\
\hline $\begin{array}{c}\text { Rarament } \\
\text { e } \\
\text { frecuente }\end{array}$ & 1 & $\begin{array}{l}\text { Para eventos que se presentan con casi nula frecuencia, y las } \\
\text { probabilidades de que se presenta son muy limitadas. }\end{array}$ \\
\hline $\begin{array}{c}\text { Poco } \\
\text { frecuente }\end{array}$ & 2 & $\begin{array}{l}\text { Existe poca probabilidad de que se presente un evento en ese } \\
\text { factor, pero en el pasado ha ocurrido en pocas ocasiones. }\end{array}$ \\
\hline Frecuente & 3 & $\begin{array}{l}\text { El evento se ha presentado en varias ocasiones y se espera que } \\
\text { pueda volver a ocurrir en algún momento. }\end{array}$ \\
$\begin{array}{c}\text { Muy } \\
\text { frecuente }\end{array}$ & 4 & $\begin{array}{l}\text { El evento sucede con mucha regularidad, las expectativas de } \\
\text { ocurrencia son altas. }\end{array}$ \\
\hline $\begin{array}{c}\text { Extremada } \\
\text { mente } \\
\text { frecuente }\end{array}$ & 5 & $\begin{array}{l}\text { El evento que afecta determinado factor ocurre con demasiada } \\
\text { frecuencia y es altamente esperado por el productor. }\end{array}$ \\
\hline
\end{tabular}

Fuente: Quirós y Solórzano (2018), con base en Palma (2011).

El impacto representa la consecuencia y potencia de afectación, se responde de acuerdo al criterio del evaluador o de la experiencia del productor, los valores establecidos para determinarlo son los siguientes:

Cuadro 2. Clasificación del impacto generado por eventos en sistemas de producción agrícol

\begin{tabular}{ccl}
$\begin{array}{c}\text { Grado de } \\
\text { impacto }\end{array}$ & $\begin{array}{c}\text { Valor } \\
\text { representado }\end{array}$ & \multicolumn{1}{c}{ Descripción de la medición } \\
\hline Insignificante & 1 & $\begin{array}{l}\text { El evento una vez materializado presenta una } \\
\text { consecuencia potencial insignificante dentro del sistema } \\
\text { y su afectación se estima casi nula. }\end{array}$ \\
\hline Leve & 2 & $\begin{array}{l}\text { El evento podria ocasionar una consecuencia potencial } \\
\text { leve dentro del sistema, pero no proporciona daños } \\
\text { significativos dentro de la producción, su efecto en el } \\
\text { rendimiento no es tan marcado. }\end{array}$ \\
\hline Moderado & 3 & $\begin{array}{l}\text { El evento podria ocasionar un impacto moderado, que } \\
\text { no representa un daño critico a la producción, pero } \\
\text { afecta de manera importante el rendimiento. }\end{array}$ \\
\hline Mrave & 4 & $\begin{array}{l}\text { La consecuencia potencial sobre la producción es grave, } \\
\text { y la perdida en el rendimiento es muy alta, afectando } \\
\text { gran parte de la producción. }\end{array}$ \\
\hline La consecuencia del evento es de magnitudes \\
catastróficas y puede representar una pérdida total o \\
casi total de la producción.
\end{tabular}

Fuente: Quirós y Solórzano (2018), con base en Palma (2011). 
El siguiente paso es asignar el grado de exposición del sistema ante el riesgo evaluado, la exposición se define como el nivel de vulnerabilidad dentro del sistema en relación con las medidas de contingencia establecidas por el productor, para hacer frente a los distintos fenómenos que se pueden presentar:

Cuadro 3. Clasificación de la exposición al riesgo en sistemas de producción agrícola.

\begin{tabular}{|c|c|c|}
\hline $\begin{array}{l}\text { Grado de } \\
\text { exposición }\end{array}$ & $\begin{array}{c}\text { Valor } \\
\text { representado }\end{array}$ & Descripción de la medición \\
\hline $\begin{array}{l}\text { Exposición } \\
\text { casi nula }\end{array}$ & 1 & $\begin{array}{l}\text { Existen amplios controles y procedimientos exhaustivos } \\
\text { para mitigar o hacer frente a la afectación que pueda } \\
\text { ocasionar el evento, en caso de que se presente el riesgo } \\
\text { las pérdidas en los rendimientos son minimos o nulos } \\
\text { dadas las medias tomadas para controlar la afectación. }\end{array}$ \\
\hline $\begin{array}{c}\text { Poco } \\
\text { expuesto }\end{array}$ & 2 & $\begin{array}{l}\text { Las medidas establecidas y los controles son amplios } \\
\text { para mitigar el riesgo, cuando se materializa el riesgo no } \\
\text { existe gran peligro de pérdida de la producción dadas las } \\
\text { medidas adoptadas }\end{array}$ \\
\hline $\begin{array}{l}\text { Exposición } \\
\text { moderada }\end{array}$ & 3 & $\begin{array}{l}\text { Existen medidas y controles establecidos pero los } \\
\text { mismos no se encuentra actualizados, puede que el } \\
\text { personal no se encuentre capacitado para las acciones a } \\
\text { tomar o bien el riesgo haya sido parcialmente mitigado } \\
\text { por estas acciones; las afectaciones en la producción } \\
\text { pueden ser notables. }\end{array}$ \\
\hline $\begin{array}{l}\text { Exposición } \\
\text { grave }\end{array}$ & 4 & $\begin{array}{l}\text { Existe escases de procedimientos o medidas para mitigar, } \\
\text { soportar o transferir los efectos del riesgo, los controles } \\
\text { existentes están desactualizados o los protocolos de } \\
\text { ejecución no se encuentran en conocimiento de los } \\
\text { trabajadores, una vez materializado el riesgo la afectación } \\
\text { en la producción es importante y los rendimientos se } \\
\text { reducen notablemente. }\end{array}$ \\
\hline $\begin{array}{l}\text { Gravemente } \\
\text { expuesto }\end{array}$ & 5 & $\begin{array}{l}\text { Los procedimientos son nulos o casi inexistentes, no hay } \\
\text { personas definidas para llevar a cabo las acciones } \\
\text { necesarias y los riesgos no fueron transferidos a terceros, } \\
\text { en caso de materializarse el riesgo la afectación es } \\
\text { importante y los rendimientos se ven seriamente } \\
\text { afectados. }\end{array}$ \\
\hline
\end{tabular}

Fuente: Quirós y Solórzano (2018), con base en Palma (2011). 
Posteriormente se debe determinar la categoría de riesgo presente para cada factor evaluado de acuerdo con la matriz de riesgo operacional que se presenta a continuación, la misma evalúa con base en los elementos anteriores la frecuencia de ocurrencia de cada evento identificado por el impacto en los rendimientos, si se utiliza una escala del 1 al 5, se presentan los rangos del resultado de la multiplicación de la frecuencia por su impacto, a su vez que evalúa las medidas tomadas por el agricultor para gestionar dicho riesgo en el apartado de exposición.

Cuadro 4. Matriz de riesgo operacional en sistema de producción agrícola
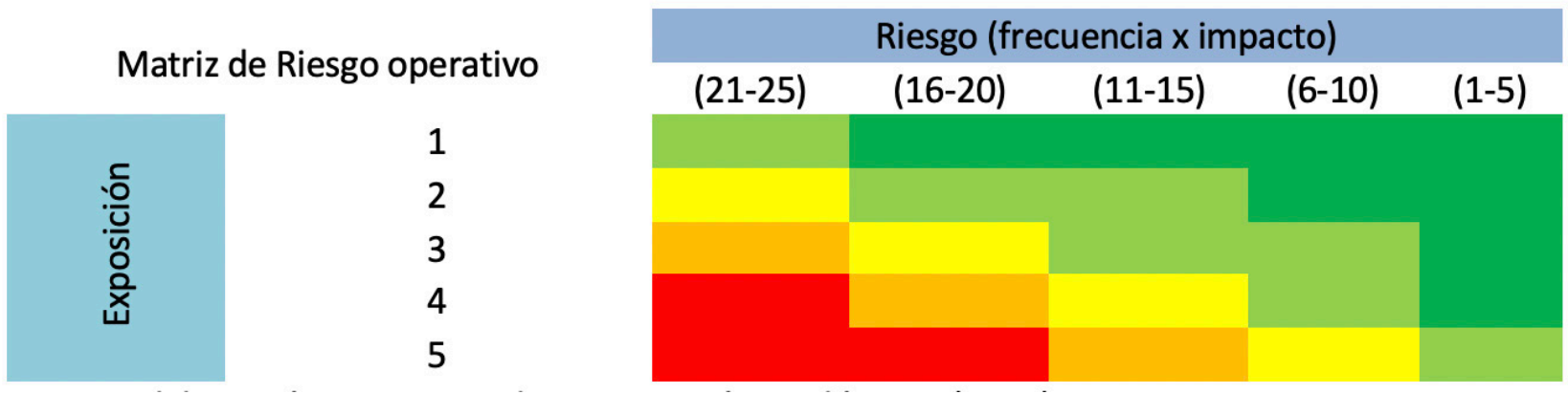

Fuente: Elaboración propia, con base en Quirós y Solórzano (2018).

Cada casilla de la matriz de riesgo representa un grado distinto de acuerdo a la evaluación de cada factor para cada fuente de riesgo y de acuerdo a la exposición (gestión) que presenta el productor, es decir, que tan factibles son las medidas que adopta, si es que las posee, para auto asegurar su producción, mitigar o reducir el impacto de los riesgos presentes en su sistema productivo. Así mismo, la línea de color produce un efecto semáforo de advertencia para priorizar aquellos eventos con mayor relevancia, los rangos de estos colores se pueden establecer de acuerdo a los criterios del evaluador según su efecto en la producción.

\section{Conclusiones}

El presente documento se enfocó en la clasificación de los factores de riesgo operativo que como su concepto lo establece, delimita su evaluación en los procesos productivos, la gestión del recurso humano, la gestión de las tecnologías productivas y los factores climáticos y ambientales; dando énfasis en su afectación sobre la producción de cada sistema productivo de manera individual, es decir, parte del punto que son factores sobre los cuáles el productor puede tomar decisiones para la mitigación y o reducción del riesgo.

La importancia de determinar estos factores para la evaluación permitió estandarizar parámetros sobre los cuáles se puede realizar una medición del riesgo, aspecto que no se ha tomado en cuenta para estudios anteriores, o que, en su defecto, no se tomaba en cuenta la integrali- 
dad de un sistema agrícola.

Estos factores permiten identificar con mayor detalle y minuciosidad eventos dentro de cada uno de ellos, que representan un riesgo para la producción, ya sea por una mala gestión por parte del productor, o bien por desconocimiento de estas variables y su posible afectación en los rendimientos productivos.

Para analizar y evaluar cada factor de riesgo de manera empírica, las llamadas matrices de calor o bien matrices de riesgo operativo resultan de fácil uso y compresión, ya que es el método utilizado por entidades financieras y empresariales para evaluar riesgos, cuando no se posee información histórica sobre la cual realizar análisis de tipo estadistico, sobre los cuales se basan las principales metodologías de evaluación de riesgo.

Es importante realizar evaluaciones integrales bajo esta metodología para productores del sector agrícola, y ampliar la investigación y desarrollo del método a los diferentes ámbitos del área agropecuaria. Se debe trabajar además, en estudios delimitadores sobre la importancia relativa de cada factor, como lo establecido por Quirós y Solórzano (2018), para darle un valor agregado a esta metodología, ya que las matrices por si solas no generan índices de riesgo o indicadores sobre los cuales tomar decisiones; y que a su vez permita incorporar parámetros que ayuden a monitorear y analizar la gestión; estos pueden ser integrardos en herramientas informáticas, como lo son las aplicaciones de dispositivos móviles, para un fácil uso por parte de los agricultores.

\section{Literatura citada}

Arbeláez, J. C., Franco, L. C., Betancur, C., Murillo, J. G., Andrea, P., Henao, V. M., ... Varela, D. C. (2006). Riesgo operacional: reto actual de las entidades financieras. Revista Ingenierías Universidad de Medellín, (5), 14.

Baquet, A. E., Hambleton, R., \& Jose, D. (1997). Introduction to risk management. Recuperado de http://agris.fao.org/agris-search/search.do?recordID=US201300017609

CEPAL. (2013). Gestión integral de riesgos y seguros agropecuarios en Centroamérica y la República Dominicana: situación actual y líneas de acción potenciales. CEPAL. Recuperado de: https://www.cepal.org/es/publicaciones/27170-gestion-integral-riesgos-seguros-agropecuarios-centroamerica-la-republica

Delfiner, M., \& Pailhé, C. (2009). Técnicas cualitativas para la gestión del Riesgo Operacional [MPRA Paper]. Recuperado el 12 de febrero de 2019, de https://mpra.ub.uni-muenchen. de/15809/

Engler, A. (2009). Estrategias del Manejo de riesgo. En C. Ruiz, Gestión del riesgo agropecuario. Boletín INIA N 186. . Instituto de Investigaciones Agropecuarias (INIA) (págs. 25-40 p). Centro Regional de Investigación Quilamapu, Chillán, Chile.

Fahad, S., Wang, J., Khan, A. A., ullah, A., Ali, U., Hossain, M. S., ... Bilal, A. (2018). Evaluation of 
farmers' attitude and perception toward production risk: Lessons from Khyber Pakhtunkhwa Province, Pakistan. Human and Ecological Risk Assessment: An International Journal, 24(6), 1710-1722. https://doi.org/10.1080/10807039.2018.1460799

FAO. (2003). Agricultura Orgánica, Ambiente y Seguridad Alimentaria. Roma: FAO. Recuperado de http://www.fao.org/3/y4137s/y4137soo.htm\#Contents

FAO. (15 de marzo de 2018). Los desastres causan pérdidas agrícolas millonarias, con la sequía a la cabeza. Organización de las Naciones Unidas para la Alimentación y la Agricultura. Recuperado de http://www.fao.org/news/story/es/item/1107192/icode/

Girdžiūtè, L. (2012). Risks in Agriculture and Opportunities of their Integrated Evaluation. Procedia - Social and Behavioral Sciences, 62, 783-790. https://doi.org/10.1016/j.sbspro.2012.09.132

Guerra, G. (2002). El agronegocio y la empresa agropecuaria frente al siglo XXI. San José, Costa Rica: IICA.

Hansson, H., \& Lagerkvist, C. J. (2012). Measuring farmers' preferences for risk: a domain-specific risk preference scale. Journal of Risk Research, 15(7), 737-753. https://doi.org/10.1080/13 669877.2012.657217

Hardaker, J. B., Lien, G., Anderson, J. R., \& Huirne, R. B. M. (Eds.). (2015). Coping with risk in agriculture: applied decision analysis (за ed.). Wallingford: CABI. https://doi. org/10.1079/9781780645742.0000

Harwood, J., Heifner, R., Coble, K., Perry, J., \& Somwaru, A. (1999). Managing Risk in Farming: Concepts, Research, and Analysis. Resuperado de: https://www.researchgate.net/publication/23517002

Just, R. E., \& Pope, R. D. (2003). Agricultural Risk Analysis: Adequacy of Models, Data, and Issues. American Journal of Agricultural Economics, 85(5), 1249-1256. https://doi.org/10.1111/ j.0092-5853.2003.00538.x

Kahan, D. (2013). Managing risk in farming (Reprint). Rome: FAO. Recuperado de: http:// www.fao.org/uploads/media/3-ManagingRiskInternLores.pdf

Miller, A., Dobbins, C., Pritchett, J., Boehlje, M., \& Ehmke, C. (2004). Risk Management for farmers. Department of Agricultural Economics. Purdue University. Recuperado de: http://future. aae.wisc.edu/publications/risk_management_for_farmers.pdf.

Mishra, A. K., \& Lence, S. H. (2005). Risk management by farmers, agribusinesses, and lenders. Agricultural Finance Review, 65(2), 131-148. https://doi.org/10.1108/00214660580001169

Mora, J. A. N., \& Gudiño, J. J. C. (2010). Riesgo operativo: esquema de gestión y modelado del riesgo. Análisis Económico, XXV(58), 123-157.

Mora, A. (2014). El uso de la distribución g-h en riesgo operativo. Contaduría y Administración, 59(1), 123-148. https://doi.org/10.1016/S0186-1042(14)71246-X 
Musser, W. N., \& Patrick, G. F. (2002). How Much does Risk Really Matter to Farmers? En R. E. Just \& R. D. Pope (Eds.), A Comprehensive Assessment of the Role of Risk in U.S. Agriculture (p. 537-556). Boston, MA: Springer US. https://doi.org/10.1007/978-1-4757-3583-3_24

Odening, M., \& Shen, Z. (2014). Challenges of insuring weather risk in agriculture. Agricultural Finance Review, 74(2), 188-199. https://doi.org/10.1108/AFR-11-2013-0039

OECD. (2009). Managing Risk in Agriculture: A Holistic Approach. OECD. https://doi.org/10.1787/9789264075313-en

Pacheco, D. (2009). Riesgo Operacional: Conceptos y Mediciones. Dirección de estudios y análisis financiero. Departemento de estudios unidad de riesgos. Superintendencia de Bancos e Instituciones Financieras. Chile. Recuperado de: https://wwww.sbif.cl/sbifweb/internet/ archivos/publicacion_8511.pdf

Palma Rodriguez, C. (2011). ¿Cómo construir una matriz de riesgo operativo? Ciencias Económicas, (1), 7.

Pelka, N., Musshoff, O., \& Weber, R. (2015). Does weather matter? How rainfall affects credit risk in agricultural microfinance. Agricultural Finance Review, 75(2), 194-212. https://doi. org/10.1108/AFR-10-2014-0030

Quirós Badilla, D. F., \& Solórzano Thompson, J. (2018). Propuesta de una metodología para la evaluación de riesgo operativo en sistemas de producción agrícola en Costa Rica aplicado a un estudio de caso en el cultivo de café (Coffea arabica). (Trabajo Final de Graduación para optar por el título de Licenciatura en Economía Agrícola con énfasis en Agroambiente). Universidad de Costa Rica. San José, Costa Rica.

Seaton, R., \& Barea, L. (2013). The New Zealand falcon and wind farms: a risk assessment framework. New Zealand Journal of Zoology, 40(1), 16-27. https://doi.org/10.1080/03014223.2 012.754361

SEPSA. (21 de junio del 2019). Estadísticas Agropecuarias: Población y empleo. Recuperado de infoagro: http://Www.infoagro.go.cr/EstadisticasAgropecuarias/PoblacionEmpleo/Paginas/default.aspx\#c01

Ullah, R., Jourdain, D., Shivakoti, G. P., \& Dhakal, S. (2015). Managing catastrophic risks in agriculture: Simultaneous adoption of diversification and precautionary savings. International Journal of Disaster Risk Reduction, 12, 268-277. https://doi.org/10.1016/j.ijdrr.2015.02.001 